\title{
Theory overview of semileptonic $B$ decays
}

\section{Olcyr Sumensari*}

Istituto Nazionale Fisica Nucleare, Sezione di Padova, I-35131 Padova, Italy Dipartamento di Fisica e Astronomia "G. Galilei", Università di Padova, Italy

E-mail: olcyr.sumensari@pd.infn.it

I review the recent theoretical progress on the study of tree-level semileptonic $B$-meson decays. After briefly reviewing the latest developments on the exclusive determinations of $\left|V_{c b}\right|$, I discuss the hints of lepton flavor universality violation in charged currents. Particular emphasis is given to the implications of these anomalies and to the possibilities to experimentally test the proposed New Physics explanations.

The International Conference on B-Physics at Frontier Machines - BEAUTY2018

6-11 May, 2018

La Biodola, Elba Island, Italy

${ }^{*}$ Speaker. 


\section{Introduction}

Tree-level semileptonic decays of mesons provide an straightforward way to extract the moduli of several CKM matrix elements, which can then be confronted with the CKM matrix unitarity. In the last decade, precision studies of semileptonic $B$-meson decays have been made possible by the the large samples of $B$-mesons collected at the $B$-factories (BaBar and Belle) and LHCb. At the same time, we witnessed a huge progress of numerical simulations of QCD on the lattice (LQCD), which nowadays allow us to attain a percent level precision for certain hadronic quantities. For these reasons, semileptonic $B$ decays offer a very promising route to seek the effects of New Physics (NP).

One of the most intriguing results from the LHC is the indication of lepton flavor universality (LFU) violation, observed in both tree-level and loop-induced semileptonic $B$-decays. The $B$-factories and LHCb observed a departure from the SM in the tree-level processes, mediated by the charged currents,

$$
R_{D^{(*)}}=\frac{\mathscr{B}\left(B \rightarrow D^{(*)} \tau \bar{v}\right)}{\mathscr{B}\left(B \rightarrow D^{(*)} l \bar{v}\right)}, \quad l=e, \mu,
$$

which turned out to be $\approx 2-3 \sigma$ above the SM predictions [1-7]. When combined in the same fit, these results amount to a discrepancy with respect to the SM at the $\approx 4 \sigma$ level [8]. Furthermore, another intriguing indication of LFU violation appeared in loopinduced processes,

$$
R_{K^{(*)}}=\left.\frac{\mathscr{B}\left(B \rightarrow K^{(*)} \mu \mu\right)}{\mathscr{B}\left(B \rightarrow K^{(*)} e e\right)}\right|_{q^{2} \in\left[q_{\min }^{2}, q_{\max }^{2}\right]},
$$

measured by LHCb in different $q^{2}$-bins below the $c \bar{c}$ resonances $[9,10]$. The obtained values appear to be $\approx 2.5 \sigma$ below the $\mathrm{SM}$ predictions $[11,12]$. Both of these ratios are defined in such a way that the CKM matrix element dependence and most of the hadronic uncertainties cancel-out, providing rather clean observables. Therefore, if confirmed with more data, they will represent a very clear indication of NP.

In the following we will focus on two issues that recently attracted the attention of the theory community: (i) the extraction of $\left|V_{c b}\right|$ from $B \rightarrow D^{*} l \bar{v}$ decays, and (ii) the abovementioned hints of LFU violation in charged-current processes. ${ }^{1}$

\section{Exclusive determinations of $\left|V_{c b}\right|$ : latest developments}

The CKM matrix element $V_{c b}$ is a free parameter of the SM that plays an important role in the unitarity triangle analysis and in the prediction of flavor changing neutral currents $[14,15]$. Its value is extracted by confronting the experimental results for the processes based on the transition $b \rightarrow c l \bar{v}$ with their SM predictions. Surprisingly, the

\footnotetext{
${ }^{1}$ The reader is referred to other talks of the same conference for a discussion of the LFU anomalies in neutral currents [13].
} 
values determined by using inclusive and exclusive $B$-meson decays show a discrepancy that only grew with time. The current averages quoted by HFLAV are [8]

$$
\begin{array}{ll}
\left|V_{c b}\right|=(42.19 \pm 0.78) \times 10^{-3}, & \text { from } B \rightarrow X_{c} l \bar{v}, \\
\left|V_{c b}\right|=\left(39.05 \pm 0.47_{\exp } \pm 0.58_{\text {th }}\right) \times 10^{-3}, & \text { from } B \rightarrow D^{*} l \bar{v} \\
\left|V_{c b}\right|=\left(39.18 \pm 0.94_{\exp } \pm 0.36_{\text {th }}\right) \times 10^{-3}, & \text { from } B \rightarrow D l \bar{v},
\end{array}
$$

which differ by $\approx 3 \sigma$. To reliably extract these values it is crucial to control the hadronic uncertainties. ${ }^{2}$ While the relevant $B \rightarrow D$ form factors have been computed at nonzero recoil values by means of LQCD simulations $[16,17]$, c.f. discussion in Sec. 3.1, some pieces of information on the $B \rightarrow D^{*}$ form factors are still lacking. The usual strategy in the latter case is to employ the overall form-factor normalization $\left[h_{A_{1}}(1)\right]$ computed on the lattice $[18,19]$ and to extract their shapes from the experimental angular distributions of $B \rightarrow D^{*}(\rightarrow D \pi) l \bar{v}$, by using a convenient parameterization. The parameterization considered by the experimental collaborations was the one proposed by Caprini, Lellouch and Neubert (CLN) [20], which suggests

$$
\begin{aligned}
h_{A_{1}}(w) & =h_{A_{1}}(1)\left[1+8 \rho^{2} z+\left(53 \rho^{2}-15\right) z^{2}-\left(231 \rho^{2}-91\right) z^{3}\right], \\
R_{1}(w) & =R_{1}(1)-0.12(w-1)+0.05(w-1)^{2}, \\
R_{2}(w) & =R_{2}(1)-0.11(w-1)-0.06(w-1)^{2},
\end{aligned}
$$

where $w=\left(m_{B}^{2}+m_{D}^{2}-q^{2}\right) /\left(2 m_{B} m_{D}\right)$ is the relative velocities of the initial and final state mesons, and $z=(\sqrt{w+1}-\sqrt{2}) /(\sqrt{w+1}+\sqrt{2})$. This parameterization is based on two approximations: (i) the shape of the form factor $h_{A_{1}}(w)$, which corresponds to the Isgur-Wise function in the heavy-quark limit, is truncanted to a quadratic power of $w-1$. Unitarity and analyticity are then used to express the shape parameters in Eq. (2.4) in terms of a single parameter $\rho$, c.f. Ref. [20-22]; (ii) The ratios $R_{1,2}(w)$ between other form factors and $h_{A_{1}}(w)$ are also parameterized by a power expansion around zero-recoil values $(w=1)$, with the shape parameters in Eq. (2.5) and (2.6) fixed by heavy quark effective theory (HQET) considerations. The overall normalization $\left[h_{A_{1}}(1)\right]$ is then determined on the lattice $[18,19]$, while the three remaining parameters in (2.4)-(2.6), namely $\rho$ and $R_{1,2}(1)$, are extracted from the $B \rightarrow D^{*} l \bar{v}$ data, c.f. e.g. Ref. [8]. ${ }^{3}$

Recently, the publication of the unfolded $B \rightarrow D^{*} l \bar{v}$ Belle data [23] allowed theorists to perform their own extraction of $\left|V_{c b}\right|^{\text {excl. }}$ by using different parameterizations. Surprisingly, the authors of Ref. $[24,25]$ independently concluded that the obtained value of $\left|V_{c b}\right|^{\text {excl. }}$ is parameterization dependent. The parameterization considered in these papers was the one

\footnotetext{
${ }^{2}$ The relevant $B \rightarrow D^{(*)}$ hadronic matrix elements are expressed in terms of two (four) form factors in the SM: $\left\langle D(k)\left|\bar{c} \gamma^{\mu} P_{L} b\right| B(p)\right\rangle \propto f_{+}\left(q^{2}\right), f_{0}\left(q^{2}\right)$ and $\left\langle D^{*}(k)\left|\bar{c} \gamma^{\mu} P_{L} b\right| B(p)\right\rangle \propto V\left(q^{2}\right), A_{1}\left(q^{2}\right), A_{2}\left(q^{2}\right), A_{0}\left(q^{2}\right)$, where $q^{2}=(p-k)^{2}$ is the dilepton squared mass. Moreover, the scalar and pseudoscalar form factors, $f_{0}\left(q^{2}\right)$ and $A_{0}\left(q^{2}\right)$, only contribute significantly to $B \rightarrow D^{(*)} \tau \bar{v}$.

${ }^{3}$ It is worth to stress that the accuracy of the approximation in Eq. (2.5) and (2.6) was estimated to be better than $2 \%$ in Ref. [21]. The uncertainty associated to the shape parameters, negligible at the time, are now important and should be included in experimental analyses.
} 
named after Boyd, Grinstein and Lebed (BGL) [22], which differs from CLN by the fact that the shape parameters of $R_{1,2}(w)$ in Eq. (2.5)-(2.6) are not fixed by HQET. Instead, these are new parameters that are also extracted from experimental data. The results obtained with both parameterizations read $[24,25]$

$$
\left|V_{c b}\right|_{\mathrm{CLN}}^{\text {excl. }}=(38.2 \pm 1.5) \times 10^{-3}, \quad\left|V_{c b}\right|_{\mathrm{BGL}}^{\text {excl. }}=41.7_{-2.1}^{+2.0} \times 10^{-3}
$$

Interestingly, the values obtained with BGL are closer to the inclusive value. ${ }^{4}$. While the BGL parameterization seems to be more attractive, since it does not rely on HQET relations, we still cannot conclude that one parameterization is better than another. Both fits provide an equally good description of current data. For that reason, we still need to wait for LQCD and Belle-II data at small-recoil values, which might finally solve the $V_{c b}$ problem.

\section{Lepton flavor universality violation: $R_{D}$ and $R_{D^{*}}$}

\subsection{Current status}

We shall now discuss the hints of lepton flavor universality in tree-level charged-current $B$ decays, which are independent of the above discussion. The experimental averages of the LFUV ratios $R_{D^{(*)}}$ are given by [8]

$$
R_{D}^{\exp }=\frac{\mathscr{B}(B \rightarrow D \tau \bar{v})}{\mathscr{B}(B \rightarrow D l \bar{v})}=0.41(5), \quad R_{D^{*}}^{\exp }=\frac{\mathscr{B}\left(B \rightarrow D^{*} \tau \bar{v}\right)}{\mathscr{B}\left(B \rightarrow D^{*} l \bar{v}\right)}=0.304(15)
$$

to be compared to the SM predictions $R_{D}^{\mathrm{SM}}=0.299(7)[16,17]$ and $R_{D^{*}}^{\mathrm{SM}}=0.257(3)$ [27], which are respectively $\approx 2 \sigma$ and $\approx 3 \sigma$ larger than the experimental values given above. ${ }^{5}$ As anticipated in the introduction, the main advantage of using ratios as in the equations above is that many hadronic uncertainties actually cancel, providing theoretically clean observables. Nonetheless, there are still residual hadronic uncertainties that must be estimated. An important ingredient in the computation of $R_{D^{(*)}}$ is that the $\tau$-lepton mass is not negligible compared to $m_{B}$. Therefore, the (pseudo)scalar form factors, which do not contribute to the rates with light leptons, are needed to reliably predict these quantities. In the case of $R_{D}$ both scalar, $f_{0}\left(q^{2}\right)$, and vector, $f_{+}\left(q^{2}\right)$, form factors have been computed on the lattice in the region of large $q^{2}$ by two different collaborations $[16,17]$. Extrapolation to $q^{2}=0$ is highly constrained by the relation $f_{0}(0)=f_{+}(0)$. The situation is slightly less favorable in the case of $R_{D^{*}}$ since the needed form factors are not yet available from LQCD simulations at nonzero recoils. The strategy adopted is to extract the leading form factors from $B \rightarrow D^{*} l \bar{v}$, as explained in Sec. 2. The remaining pseudoscalar form factor $A_{0}\left(q^{2}\right)$, which cannot be extracted from data, is then obtained by obtained by considerations based on HQET with generous error bars [27,30]. The overall agreement in the literature is that the departures from the SM cannot be explained by only relying

\footnotetext{
${ }^{4}$ Consistent results have also been very recently reported by the Belle collaboration [26]

${ }^{5}$ Other predictions found in the literature are $R_{D^{*}}^{\mathrm{SM}}=0.252(3)[28], R_{D^{*}}=0.257(5)[29]$ and $R_{D^{*}}^{\mathrm{SM}}=$ $0.260(8)[30]$, in agreement with the values quoted above.
} 
on underestimated hadronic uncertainties. This conclusion is mostly based on the fact that the (unknown) $A_{0}\left(q^{2}\right)$ form factor gives a numerically subleading contribution to $R_{D^{*}}$. Nonetheless, it goes without saying that a LQCD computation of this form factor would be extremely useful. Finally, it is worth mentioning that another potential source of uncertainty comes from the soft-photon radiation, which have only been partially estimated in Ref. [31].

\subsection{Effective field theory description}

In the following, we will assume that the $R_{D^{(*)}}$ anomalies are due to NP and we will discuss their implications. The transition $b \rightarrow c \ell \bar{v}$ (with $\ell=e, \mu, \tau$ ) can be generically described at low-energies by the following effective Lagrangian, at dimension-6,

$$
\begin{aligned}
\mathscr{L}_{\text {eff }}=-2 \sqrt{2} & G_{F} V_{c b}\left[\left(1+g_{V_{L}}^{\ell}\right)\left(\bar{c}_{L} \gamma^{\mu} b_{L}\right)\left(\bar{\ell}_{L} \gamma_{\mu} v_{L}\right)+g_{V_{R}}^{\ell}\left(\bar{c}_{R} \gamma^{\mu} b_{R}\right)\left(\bar{\ell}_{L} \gamma_{\mu} v_{L}\right)\right. \\
& \left.+g_{S_{R}}^{\ell}\left(\bar{c}_{L} b_{R}\right)\left(\bar{\ell}_{R} v_{L}\right)+g_{S_{L}}^{\ell}\left(\bar{c}_{R} b_{L}\right)\left(\bar{\ell}_{R} v_{L}\right)+g_{T}^{\ell}\left(\bar{c}_{R} \sigma^{\mu v} b_{L}\right)\left(\bar{\ell}_{R} \sigma_{\mu v} v_{L}\right)\right]+ \text { h.c., }
\end{aligned}
$$

where $g_{V_{L(R)}}^{\ell}, g_{S_{L(R)}}^{\ell}$ and $g_{T}^{\ell}$ are generic Wilson coefficients. Since the NP responsible for these interaction must arise above the electro-weak scale, one should further impose $S U(2)_{L} \times$ $U(1)_{Y}$ gauge invariance to the above Lagrangian [32,33]. The operators one should then consider are [34]

$$
\begin{array}{rlrl}
{\left[\mathscr{O}_{\ell q}^{(3)}\right]_{p r s t}} & =\left(\overline{L_{p}} \gamma_{\mu} \tau^{I} L_{r}\right)\left(\overline{Q_{s}} \gamma^{\mu} \tau^{I} Q_{t}\right), & & {\left[\mathscr{O}_{\text {lequ }}^{(1)}\right]_{p r s t}=\left(\overline{L_{p}^{a}} e_{r R}\right) \varepsilon_{a b}\left(\overline{Q_{s}^{b}} u_{t R}\right),} \\
{\left[\mathscr{O}_{\text {ledq }}\right]_{p r s t}=\left(\overline{L_{p}^{a}} e_{r R}\right)\left(\overline{d_{s R}} Q_{t}^{a}\right),} & {\left[\mathscr{O}_{\text {lequ }}^{(3)}\right]_{p r s t}=\left(\overline{L_{p}^{a}} \sigma_{\mu v} e_{r R}\right) \varepsilon_{a b}\left(\overline{Q_{s}^{b}} \sigma^{\mu v} u_{R t}\right),}
\end{array}
$$

where $a, b$ are $S U(2)_{L}$ indices, $\varepsilon_{12}=-\varepsilon_{21}=1$, and $p, r, s, t$ are flavor indices. The matching at $\mu=m_{b}$ of this basis of operators onto the $S U(3)_{c} \times U(1)_{\mathrm{em}}$ invariant one, given in Eq. (3.2), tell us that $g_{V_{R}}^{\ell}$ cannot break LFU at dimension-6. This coefficient can only be generated by the LFU conserving operator $\mathscr{O}_{H u}=\left(H^{\dagger} i \overleftrightarrow{D}_{\mu} H\right)\left(\bar{u}_{p R} \gamma^{\mu} u_{r R}\right)$, being irrelevant for the following discussion [34-36]. The other Wilson coefficients have a one-to-one correspondence to the basis given above.

To identify the allowed combination of Wilson coefficients at $\mu=m_{b}$, one should perform a fit to the experimental values in Eq. (1.1). The compact expressions for these observable are given by [37]

$$
\begin{aligned}
\frac{R_{D^{(*)}}}{R_{D^{(*)}}^{\mathrm{SM}}}=\left|1+g_{V_{L}}^{\tau}\right|^{2} & +a_{S}^{D^{(*)}}\left|g_{S}^{\tau}\right|^{2}+a_{P}^{D^{(*)}}\left|g_{P}^{\tau}\right|^{2}+a_{T}^{D^{(*)}}\left|g_{T}^{\tau}\right|^{2}+a_{S V_{L}}^{D^{(*)}} \operatorname{Re}\left[g_{S}^{\tau}\left(1+\left(g_{V_{L}}^{\tau}\right)^{*}\right)\right] \\
& +a_{P V_{L}}^{D^{(*)}} \operatorname{Re}\left[g_{P}^{\tau}\left(1+\left(g_{V_{L}}^{\tau}\right)^{*}\right)\right]+a_{T V_{L}}^{D^{(*)}} \operatorname{Re}\left[g_{T}^{\tau}\left(1+\left(g_{V_{L}}^{\tau}\right)^{*}\right)\right],
\end{aligned}
$$

where $g_{S(P)}^{\tau}=g_{S_{R}}^{\tau} \pm g_{S_{L}}^{\tau}$, and the numeric coefficients $a_{i}^{D}$ and $a_{i}^{D^{*}}$ are collected in Table 1 . Interestingly, $R_{D}$ and $R_{D^{*}}$ are sensitive to a complementary set of NP operators. While $R_{D}$ is sensitive to both scalar and tensor contributions, $R_{D^{*}}$ only receives sizable contributions from the tensor ones. Furthermore, the scenario with only $g_{V_{L}}$ predicts $R_{D} / R_{D}^{\mathrm{SM}}=R_{D^{*}} / R_{D^{*}}^{\mathrm{SM}}$, 
since it corresponds to an overall shift of the SM. In this case, a viable explanation of $R_{D^{(*)}}$ can be obtained, to $1 \sigma$ accuracy, for

$$
g_{V_{L}}^{\tau} \in(0.09,0.13)
$$

Other viable solutions, which are not in the (current) $\times($ current $)$ form, are not only possible but also well motivated by concrete NP scenarios, as we will discuss in Sec. 3.3. The possibility of considering (pseudo)scalar operators (i.e. only $g_{S_{L(R)}} \neq 0$ ) was considered in the past, but it has been recently shown that such a scenario containing is in strong tension with the $\boldsymbol{B}_{c}$-meson lifetime constraint $[38,39]$. More precisely, the pseudoscalar interactions lift the helicity suppression of the SM rate for

$$
\mathscr{B}\left(B_{c} \rightarrow \tau \bar{v}\right)=\tau_{B_{c}} \frac{m_{B_{c}} f_{B_{c}}^{2} G_{F}^{2}\left|V_{c b}\right|^{2}}{8 \pi} m_{\tau}^{2}\left(1-\frac{m_{\tau}^{2}}{m_{B_{c}}^{2}}\right)^{2}\left|1+g_{P}^{\tau} \frac{m_{B_{c}}^{2}}{m_{\tau}\left(m_{b}+m_{c}\right)}\right|^{2},
$$

where $f_{B_{c}}=427(6) \mathrm{MeV}$ is the $B_{c}$-meson decay constant [40]. The current experimental value on $\tau_{B_{c}}=0.507(9) \times 10^{-12} \mathrm{~s}$ allows us to set a conservative limit of $30 \%$ on $\mathscr{B}\left(B_{c} \rightarrow\right.$ $\tau \bar{v})$ [38], which can then be translated onto the $1 \sigma$ bound, $g_{P}^{\tau}\left(\mu=m_{b}\right) \in(-1.14,0.68)$, where we have used $\left|V_{c b}\right|=0.0417(20)[24,25] .{ }^{6}$ This constraint is illustrated in the left panel of Fig. 1, where it can be seen that an explanation of $R_{D^{(*)}}$ via only (pseudo)scalar operators is ruled out. Scenarios providing a good fit to $R_{D^{(*)}}$, while avoiding the $B_{c^{-}}$-lifetime constraint, can be obtained by using a different set of operators. For instance, a scenario containing only $g_{S_{L}}$ and $g_{T}$ can provide a good description of current data, as illustrated in the right panel of Fig. 1. This combination of Wilson coefficient is well motivated by scenarios containing lepto-quark bosons which predict certain correlations between $g_{S_{L}}$ and $g_{T}$. Furthermore, it is known that a non-negligible mixing of $g_{T}$ into $g_{S_{L}}$ is induced via electroweak quantum corrections [42], c.f. also Ref. [37]. Finally, note that viable scenarios with complex Wilson coefficients have also been considered in the literature, providing an equally good fit to the experimental results Ref. [44-47].

\begin{tabular}{|c|cccccc|}
\hline Decay mode & $a_{S}^{M}$ & $a_{S V_{L}}^{M}$ & $a_{P}^{M}$ & $a_{P V_{L}}^{M}$ & $a_{T}^{M}$ & $a_{T V_{L}}^{M}$ \\
\hline \hline$B \rightarrow D$ & $1.08(1)$ & $1.54(2)$ & 0 & 0 & $0.83(5)$ & $1.09(3)$ \\
$B \rightarrow D^{*}$ & 0 & 0 & $0.0473(5)$ & $0.14(2)$ & $17.3(16)$ & $-5.1(4)$ \\
\hline
\end{tabular}

Table 1: Numeric coefficients in Eq. (3.4) for $M=D, D^{*}$ obtained in Ref. [37] by assuming that NP only modifies the coefficients $g_{V_{L}}, g_{S_{L(R)}}$ and $g_{T}$ for the transition $b \rightarrow c \tau \bar{v}$.

\subsection{Concrete New Physics scenarios}

Several concrete models of NP can generate the viable Wilson coefficients discussed above. These scenarios require new bosonic degrees of freedom at the TeV scale [48] with

\footnotetext{
${ }^{6}$ Alternatively, one could consider the less conservative limit $\mathscr{B}\left(B_{c} \rightarrow \tau \bar{v}\right) \lesssim 10 \%$ computed in Ref. [41]. By using this constraint instead, the bound would become $g_{P} \in(-0.76,0.30)$ to $1 \sigma$ accuracy.
} 

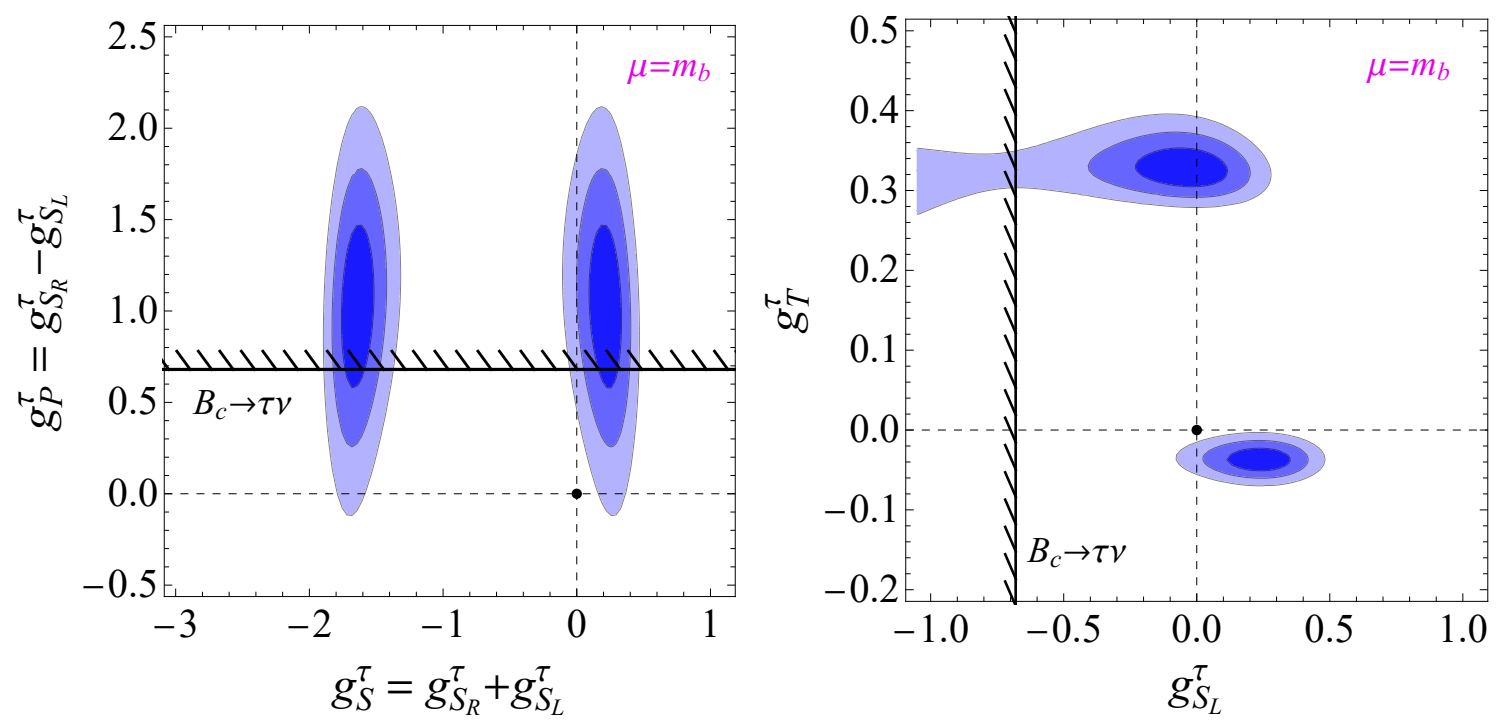

Figure 1: Allowed regions by $R_{D}$ and $R_{D^{*}}$ in the planes $g_{S}^{\tau}$ vs. $g_{P}^{\tau}$ (left panel), and $g_{S_{L}}^{\tau}$ vs. $g_{T}^{\tau}$ (right panel) are shown to 1, 2 and $3 \sigma$ accuracy in blue (darker to lighter). The black lines show the constraints from the $B_{c}$-meson lifetime, which allow us to exclude the solutions with large values of $\left|g_{P}\right|$, as explained in the text [37].

tree-level contributions to the transition $b \rightarrow c \tau \bar{v}$. The main challenge is to comply with nontrivial constraints coming flavor physics observables, electroweak precision tests [49-51] and LHC searches [52]. The proposed mediators can be (i) a charged Higgs, (ii) colorless vector mesons $\left(W^{\prime}\right)$, and (iii) scalar or vector leptoquarks (LQ). Scenarios with charged Higgs boson cannot accommodate $R_{D^{(*)}}$ due to the $B_{c^{-}}$-lifetime constraint discussed above, c.f. Fig. 1. On the other hand, $W^{\prime}$ models have difficulties to evade nontrivial LHC constraints on di-tau production [52-54]. ${ }^{7}$ The only minimalistic scenarios capable of explaining $R_{D^{(*)}}$ while satisfying other existing constraints are LQs.

In Table 2 we list the LQ states that can contribute to $b \rightarrow c \tau \bar{v}$ in terms of their SM quantum numbers, $\left(S U(3)_{c}, S U(2)_{L}, Y\right)$, with $Q=Y+T_{3}$ [57]. From this Table we learn that very few possibilities are still viable:

- The scalar LQ $S_{1}=(\overline{\mathbf{3}}, \mathbf{1}, 1 / 3)$ generates, at the matching scale, the effective coefficients $C_{\ell q}^{(3)}$ and $C_{\ell \text { equ }}^{(1)}=-4 C_{\ell \text { equ }}^{(3)}$, which induce nonzero values of $g_{V_{L}}$ and $g_{S_{L}} \approx-8.1 g_{T}$ at $\mu=m_{b}$, after accounting for RGE effects. Both of these Wilson coefficients can accommodate for $R_{D^{(*)}}$, as discussed in Sec. 3.2. See also Ref. $[44,46,58,59]$.

- The scalar LQ $R_{2}=(\mathbf{3}, \mathbf{2}, 7 / 6)$ produces $C_{\text {lequ }}^{(1)}=4 C_{\text {lequ }}^{(3)}$, which implies $g_{S_{L}} \approx 8.1 g_{T}$ at $\mu=m_{b}$. Note that this particularly correlation of scalar and tensor coefficients lies outside of the allowed regions in Fig. 1, where real coefficients were consid-

${ }^{7}$ These constraints can be avoided by considering a $W^{\prime}$ with couplings to right-handed neutrinos [55]. However, in that case the interference term with the SM rate is not present, requiring rather large NP couplings. See also Ref. [56] for a similar proposal with scalar LQs. 
ered. However, this problem can be overcome by considering complex Wilson coefficients [44-47].

- The vector LQ $U_{1}=(\mathbf{3}, \mathbf{1}, 2 / 3)$ induces $C_{\ell q}^{(3)}$, which implies nonzero values of $g_{V_{L}}$ and $g_{S_{R}}[54]$.

Note that some of the models which predict $g_{V_{L}}^{\ell}$ in Table 2 cannot accommodate $R_{D^{(*)}}$, as shown in Table 2. This is the case because $g_{V_{L}}^{\tau}$ is constrained to be negative in these scenarios, providing a interference term with the wrong sign for $R_{D^{(*)}}$, c.f. Eq. (3.5) [60]. Furthermore, a peculiarity of the viable models listed above is a different pattern of effective operators which imply very distinctive phenomenological implications, as we will discuss in Section 3.4.

Finally, a natural question is if the hint of New Physics in $R_{K^{(*)}}^{\exp }<R_{K^{(*)}}^{\mathrm{SM}}$ and $R_{D^{(*)}}^{\exp }>R_{D^{(*)}}^{\mathrm{SM}}$ can be simultaneously explained by the same NP scenario. Building such a model turns out be a challenging task. The only mediator that can account for both anomalies at tree-level is the vector LQ $U_{1}$ [54]. The problem in this case is that model is nonrenormalible and an explicit UV-completion needs to be specified. This issue has been addressed in a series of papers with gauged models [61-65]. A more minimalistic approach, in terms of number of parameters, is to consider models containing two scalar LQs. These particles could arise, for instance, from SU(5) GUT [47] or composite Higgs models [59].

\begin{tabular}{|c|c|c|cccc||c|}
\hline Field & Spin & Quantum Numbers & $g_{V_{L}}$ & $g_{S_{R}}$ & $g_{S_{L}}$ & $g_{T}$ & $R_{D^{(*)}}$ \\
\hline \hline$R_{2}$ & 0 & $(\mathbf{3}, \mathbf{2}, 7 / 6)$ & - & - & $\checkmark$ & $\checkmark$ & $\checkmark$ \\
$S_{1}$ & 0 & $(\overline{\mathbf{3}}, \mathbf{1}, 1 / 3)$ & $\checkmark$ & - & $\checkmark$ & $\checkmark$ & $\checkmark$ \\
$S_{3}$ & 0 & $(\mathbf{3}, \mathbf{2}, 7 / 6)$ & $\checkmark$ & - & - & - & $x$ \\
\hline$V_{2}$ & 1 & $(\overline{\mathbf{3}}, \mathbf{2}, 5 / 6)$ & - & $\checkmark$ & - & - & $x$ \\
$U_{1}$ & 1 & $(\mathbf{3}, \mathbf{1}, 2 / 3)$ & $\checkmark$ & $\checkmark$ & - & - & $\checkmark$ \\
$U_{3}$ & 1 & $(\mathbf{3}, \mathbf{3}, 2 / 3)$ & $\checkmark$ & - & - & - & $x$ \\
\hline
\end{tabular}

Table 2: List of $L Q$ states contributing to the transition $b \rightarrow c \ell \bar{v}$ in terms of the SM quantum numbers, $\left(S U(3)_{c}, S U(2)_{L}, Y\right)$, and corresponding Wilson coefficients generated in Eq. (3.2). The last column summarizes the models which can accommodate $R_{D^{(*)}}$ while complying with other existing constraints. See text for details.

\subsection{Phenomenological implications}

We conclude by discussing the phenomenological implications of $R_{D^{(*)}}$ and the strategies to disentangle the viable NP explanations, if the anomalies persist. A first possibility is to study LFUV ratios of other decay modes based on the transition $b \rightarrow c \ell \bar{v}$, such as $B_{s} \rightarrow D_{s}^{(*)} \tau \bar{v}$ and $B_{c} \rightarrow J / \psi \tau \bar{v}$. In particular, LHCb performed a first measurement of the 
ratio $[66]$

$$
R_{J / \psi}=\frac{\mathscr{B}\left(B_{c} \rightarrow J / \psi \tau \bar{v}\right)}{\mathscr{B}\left(B_{c} \rightarrow J / \psi \mu \bar{v}\right)}=0.71 \pm 0.17 \pm 0.18
$$

which turns out to be again $\approx 2 \sigma$ larger than the SM estimate, $R_{J / \psi}^{\mathrm{SM}}=0.23(1)$, obtained by combining LQCD and QCD sum rules results [67]. These independent measurements can be useful not only to corroborate/refute the excess found on $R_{D^{(*)}}$, but also to provide a complementarity information about NP. For instance, as discussed in Sec. 3.2, NP in the form of (current) $\times($ current $)$ operators predict the following equality,

$$
\frac{R_{D}}{R_{D}^{\mathrm{SM}}}=\frac{R_{D^{*}}}{R_{D^{*}}^{\mathrm{SM}}}=\frac{R_{J / \psi}}{R_{J / \psi}^{\mathrm{SM}}}=\ldots,
$$

for all decay modes based on the same transition. On the other hand, scalar and/or tensor operators induce a distinct correlation between $P \rightarrow P^{\prime}$ and $P \rightarrow V$ decays, where $P^{(\prime)}$ and $V$ are generic pseudoscalar and vector mesons, respectively. Another possibility proposed in the literature is to consider the angular observables of the decay $B \rightarrow D^{*}(\rightarrow D \pi) \tau \bar{\nu}$, which have different sensitivities to the operators in Eq. (3.2) $[68,69]$. Note, in particular, that a first measurement of the $\tau$-lepton polarization asymmetry in $B \rightarrow D^{*} \tau \bar{v}$ has been made by Belle, with large error bars [5]. This observable is particularly sensitive to pseudoscalar and tensor contributions, but it is unaffected by interaction of the type $(V-A) \times(V-A)$ [70].

These semileptonic observables described above offer an alternative test to the enhancement in $R_{D^{(*)}}$, without any assumption regarding the underlying NP structure. Model independent tests can also be obtained by using purely leptonic observables. As already anticipated in Sec. 3.2, the interactions responsibles for the anomalies in $R_{D^{(*)}}$ must respect $S U(2)_{L} \times U(1)_{Y}$ gauge invariance. Writing the operators as in Eq. (3.3), at a scale $\mu \gg v_{\mathrm{EW}}$, opens the possibility to study the impact of electroweak corrections within the EFT context. It has been shown in Ref. [49-51] that the operators generating the (current) $\times($ current) contributions induce sizable corrections to $Z$-pole observables and to LFU tests in $\tau$-decays. On the other hand, the operators generating (pseudo)scalar and tensor contributions have the peculiarity of inducing chirality-enhanced contributions to $\mathscr{B}(h \rightarrow \tau \tau)$ and to the anomalous magnetic moment of the tau, respectively, without inducing any sizable modification of $Z$-pole observables and LFU tests in $\tau$-decays [37]. The ongoing experimental effort at Belle-II and LHC will offer the opportunity to check for additional enhancements in these observables. Finally, model-dependent predictions can also be obtained once a specific scenario is specified. These include signals at the LHC [52] and many other flavor observables, such as lepton flavor violating decays, c.f. e.g. Ref. [71-73]. I refer the reader to other talks in this conference covering many of these aspects [13].

\section{Summary and perspectives}

In this proceedings we discussed the recent theoretical progress on the study semileptonic $B$-meson decays. The focus of our discussion was (i) the $B \rightarrow D^{*}$ form factor parameterization dependence on the extraction of $\left|V_{c b}\right|^{\text {excl. }}$, and especially (ii) the very intriguing hints of LFU in charged currents: 
- While the recent reanalysis of $B \rightarrow D^{*} l \bar{v}$ data from Belle pointed out underestimated systematic uncertainties in the extraction of $\left|V_{c b}\right|^{\text {excl. }}[24,25]$, the $V_{c b}$ puzzle still remains since both form-factor parameterizations provide an equally good description of current data. The solution to this puzzle will come in the future with Belle-II and LQCD data on the slope of the form-factors near zero recoil.

- After summarizing the status of SM predictions, we discussed the implications of the LFU violation observed in $R_{D^{(*)}}$. By using an EFT description we argued that the operator of type $(V-A) \times(V-A)$ and the tensor one can accommodate current discrepancies, while the purely scalar ones cannot. These scenarios can be experimentally tested (i) by measuring similar LFU ratios based on the same transition, (ii) by studying the angular asymmetries of $B \rightarrow D^{(*)} \tau \bar{v}$ decays, and (iii) by studying purely leptonic observables, which are related to the LFU breaking effects via RGE effects. We have also argued that LQ bosons are the best candidates to explain these anomalies and we summarized the viable proposals made in the literature.

\section{Acknowledgments}

I would like to thank the organizers for the invitation and especially to Damir Bečirević, Svjetlana Fajfer, Ferruccio Feruglio, Nejc Košnik and Paride Paradisi from whom I learned so much about the subject of this talk. This project has received support by the European Union's Horizon 2020 research and innovation programme under the Marie SklodowskaCurie grant agreement $\mathrm{N}^{\circ} 674896$.

\section{References}

[1] J. P. Lees et al. [BaBar Collaboration], Phys. Rev. Lett. 109, 101802 (2012) [arXiv:1205.5442 [hep-ex]].

[2] J. P. Lees et al. [BaBar Collaboration], Phys. Rev. D 88, no. 7, 072012 (2013) [arXiv:1303.0571 [hep-ex]].

[3] M. Huschle et al. [Belle Collaboration], Phys. Rev. D 92, no. 7, 072014 (2015) doi:10.1103/PhysRevD.92.072014 [arXiv:1507.03233 [hep-ex]].

[4] R. Aaij et al. [LHCb Collaboration], Phys. Rev. Lett. 115, no. 11, 111803 (2015) Erratum: [Phys. Rev. Lett. 115, no. 15, 159901 (2015)] [arXiv:1506.08614 [hep-ex]].

[5] S. Hirose et al. [Belle Collaboration], Phys. Rev. Lett. 118, no. 21, 211801 (2017) [arXiv:1612.00529 [hep-ex]].

[6] Y. Sato et al. [Belle Collaboration], Phys. Rev. D 94, no. 7, 072007 (2016) [arXiv:1607.07923 [hep-ex]].

[7] A. Abdesselam et al. [Belle Collaboration], arXiv:1603.06711 [hep-ex].

[8] Y. Amhis et al. [HFLAV Collaboration], Eur. Phys. J. C 77, no. 12, 895 (2017) [arXiv:1612.07233 [hep-ex]].

[9] R. Aaij et al. [LHCb Collaboration], Phys. Rev. Lett. 113, 151601 (2014) [arXiv:1406.6482 [hep-ex]]. 
[10] R. Aaij et al. [LHCb Collaboration], JHEP 1708, 055 (2017) [arXiv:1705.05802 [hep-ex]].

[11] G. Hiller and F. Kruger, Phys. Rev. D 69 (2004) 074020 [hep-ph/0310219].

[12] M. Bordone, G. Isidori and A. Pattori, Eur. Phys. J. C 76 (2016) no.8, 440 [arXiv:1605.07633 [hep-ph]].

[13] F. Feruglio, "B-anomalies related to leptons and LFV: new directions in model building"; A. Greljo, Flavor at high-p $p_{T}$; Nazila Mahmoudi, "BSM fits for rare B decays (theory)"; M. Neubert, "Theory in the LHC era"; A. Strumia, Beyond the SM (theory); talks presented at this conference.

[14] J. Charles et al., Phys. Rev. D 91, no. 7, 073007 (2015) [arXiv:1501.05013 [hep-ph]].

[15] M. Bona et al. [UTfit Collaboration], JHEP 0610 (2006) 081 [hep-ph/0606167].

[16] H. Na et al. [HPQCD Collaboration], Phys. Rev. D 92, no. 5, 054510 (2015) Erratum: [Phys. Rev. D 93, no. 11, 119906 (2016)] [arXiv:1505.03925 [hep-lat]].

[17] J. A. Bailey et al. [MILC Collaboration], Phys. Rev. D 92, no. 3, 034506 (2015) [arXiv:1503.07237 [hep-lat]].

[18] J. A. Bailey et al. [Fermilab Lattice and MILC Collaborations], Phys. Rev. D 89, no. 11, 114504 (2014) [arXiv:1403.0635 [hep-lat]].

[19] J. Harrison, C. Davies and M. Wingate, PoS LATTICE 2016, 287 (2017) [arXiv:1612.06716 [hep-lat]].

[20] I. Caprini, L. Lellouch and M. Neubert, Nucl. Phys. B 530, 153 (1998) [hep-ph/9712417].

[21] I. Caprini and M. Neubert, Phys. Lett. B 380, 376 (1996) [hep-ph/9603414].

[22] C. G. Boyd, B. Grinstein and R. F. Lebed, Phys. Rev. D 56, 6895 (1997) [hep-ph/9705252].

[23] A. Abdesselam et al. [Belle Collaboration], arXiv:1702.01521 [hep-ex].

[24] D. Bigi, P. Gambino and S. Schacht, Phys. Lett. B 769, 441 (2017) [arXiv:1703.06124 [hep-ph]].

[25] B. Grinstein and A. Kobach, Phys. Lett. B 771, 359 (2017) [arXiv:1703.08170 [hep-ph]].

[26] E. Waheed, ICHEP 2018. https://indico.cern.ch/event/686555/contributions/2982895/.

[27] F. U. Bernlochner, Z. Ligeti, M. Papucci and D. J. Robinson, Phys. Rev. D 95, no. 11, 115008 (2017) Erratum: [Phys. Rev. D 97, no. 5, 059902 (2018)] [arXiv:1703.05330 [hep-ph]].

[28] S. Fajfer, J. F. Kamenik and I. Nisandzic, Phys. Rev. D 85, 094025 (2012) [arXiv:1203.2654 [hep-ph]].

[29] S. Jaiswal, S. Nandi and S. K. Patra, JHEP 1712, 060 (2017) [arXiv:1707.09977 [hep-ph]].

[30] D. Bigi, P. Gambino and S. Schacht, JHEP 1711, 061 (2017) [arXiv:1707.09509 [hep-ph]].

[31] D. Becirevic and N. Kosnik, Acta Phys. Polon. Supp. 3 (2010) 207 [arXiv:0910.5031 [hep-ph]]; S. de Boer, T. Kitahara and I. Nisandzic, Phys. Rev. Lett. 120 (2018) no.26, 261804 [arXiv:1803.05881 [hep-ph]].

[32] W. Buchmuller and D. Wyler, Nucl. Phys. B 268, 621 (1986).

[33] B. Grzadkowski, M. Iskrzynski, M. Misiak and J. Rosiek, JHEP 1010, 085 (2010) [arXiv:1008.4884 [hep-ph]]. 
[34] J. Aebischer, A. Crivellin, M. Fael and C. Greub, JHEP 1605, 037 (2016) [arXiv:1512.02830 [hep-ph]].

[35] S. Fajfer, J. F. Kamenik, I. Nisandzic and J. Zupan, Phys. Rev. Lett. 109, 161801 (2012) [arXiv:1206.1872 [hep-ph]].

[36] V. Bernard, M. Oertel, E. Passemar and J. Stern, Phys. Lett. B 638, 480 (2006) [hep-ph/0603202].

[37] F. Feruglio, P. Paradisi and O. Sumensari, arXiv:1806.10155 [hep-ph].

[38] R. Alonso, B. Grinstein and J. Martin Camalich, Phys. Rev. Lett. 118, no. 8, 081802 (2017) [arXiv:1611.06676 [hep-ph]].

[39] A. Celis, M. Jung, X. Q. Li and A. Pich, Phys. Lett. B 771, 168 (2017) [arXiv:1612.07757 [hep-ph]].

[40] C. McNeile, C. T. H. Davies, E. Follana, K. Hornbostel and G. P. Lepage, Phys. Rev. D 86, 074503 (2012) [arXiv:1207.0994 [hep-lat]].

[41] A. G. Akeroyd and C. H. Chen, Phys. Rev. D 96, no. 7, 075011 (2017) [arXiv:1708.04072 [hep-ph]].

[42] M. González-Alonso, J. Martin Camalich and K. Mimouni, Phys. Lett. B 772, 777 (2017) [arXiv:1706.00410 [hep-ph]].

[43] D. Bečirević, N. Košnik, O. Sumensari and R. Zukanovich Funchal, JHEP 1611, 035 (2016) [arXiv:1608.07583 [hep-ph]].

[44] Y. Sakaki, M. Tanaka, A. Tayduganov and R. Watanabe, Phys. Rev. D 88, no. 9, 094012 (2013) [arXiv:1309.0301 [hep-ph]].

[45] G. Hiller, D. Loose and K. Schönwald, JHEP 1612, 027 (2016) [arXiv:1609.08895 [hep-ph]].

[46] D. Bečirević, B. Panes, O. Sumensari and R. Zukanovich Funchal, JHEP 1806, 032 (2018) [arXiv:1803.10112 [hep-ph]].

[47] D. Bečirević, I. Doršner, S. Fajfer, D. A. Faroughy, N. Košnik and O. Sumensari, arXiv:1806.05689 [hep-ph].

[48] L. Di Luzio and M. Nardecchia, Eur. Phys. J. C 77, no. 8, 536 (2017) [arXiv:1706.01868 [hep-ph]].

[49] F. Feruglio, P. Paradisi and A. Pattori, Phys. Rev. Lett. 118, no. 1, 011801 (2017) [arXiv:1606.00524 [hep-ph]].

[50] F. Feruglio, P. Paradisi and A. Pattori, JHEP 1709, 061 (2017) [arXiv:1705.00929 [hep-ph]].

[51] C. Cornella, F. Feruglio and P. Paradisi, arXiv:1803.00945 [hep-ph].

[52] D. A. Faroughy, A. Greljo and J. F. Kamenik, Phys. Lett. B 764, 126 (2017) [arXiv:1609.07138 [hep-ph]].

[53] A. Greljo, G. Isidori and D. Marzocca, JHEP 1507, 142 (2015) [arXiv:1506.01705 [hep-ph]].

[54] D. Buttazzo, A. Greljo, G. Isidori and D. Marzocca, JHEP 1711, 044 (2017) [arXiv:1706.07808 [hep-ph]].

[55] A. Greljo, D. J. Robinson, B. Shakya and J. Zupan, arXiv:1804.04642 [hep-ph]. 
[56] D. Bečirević, S. Fajfer, N. Košnik and O. Sumensari, Phys. Rev. D 94, no. 11, 115021 (2016) [arXiv:1608.08501 [hep-ph]].

[57] I. Doršner, S. Fajfer, A. Greljo, J. F. Kamenik and N. Košnik, Phys. Rept. 641, 1 (2016) [arXiv:1603.04993 [hep-ph]].

[58] M. Bauer and M. Neubert, Phys. Rev. Lett. 116, no. 14, 141802 (2016) [arXiv:1511.01900 [hep-ph]].

[59] D. Marzocca, arXiv:1803.10972 [hep-ph].

[60] A. Angelescu, D. Becirevic, D. Faroughy and O. Sumensari, in preparation.

[61] N. Assad, B. Fornal and B. Grinstein, Phys. Lett. B 777, 324 (2018) [arXiv:1708.06350 [hep-ph]].

[62] L. Di Luzio, A. Greljo and M. Nardecchia, Phys. Rev. D 96, no. 11, 115011 (2017) [arXiv:1708.08450 [hep-ph]].

[63] M. Bordone, C. Cornella, J. Fuentes-Martin and G. Isidori, Phys. Lett. B 779, 317 (2018) [arXiv:1712.01368 [hep-ph]].

[64] R. Barbieri and A. Tesi, Eur. Phys. J. C 78, no. 3, 193 (2018) [arXiv:1712.06844 [hep-ph]].

[65] M. Blanke and A. Crivellin, Phys. Rev. Lett. 121, no. 1, 011801 (2018) [arXiv:1801.07256 [hep-ph]].

[66] R. Aaij et al. [LHCb Collaboration], Phys. Rev. Lett. 120, no. 12, 121801 (2018) [arXiv:1711.05623 [hep-ex]].

[67] D. Becirevic, D. Leljak, B. Melic and O. Sumensari, in preparation; B. Colquhoun et al. [HPQCD Collaboration], PoS LATTICE 2016 (2016) 281 [arXiv:1611.01987 [hep-lat]].

[68] D. Becirevic, S. Fajfer, I. Nisandzic and A. Tayduganov, arXiv:1602.03030 [hep-ph].

[69] M. A. Ivanov, J. G. Körner and C. T. Tran, Phys. Rev. D 95, no. 3, 036021 (2017) [arXiv:1701.02937 [hep-ph]].

[70] A. Azatov, D. Bardhan, D. Ghosh, F. Sgarlata and E. Venturini, arXiv:1805.03209 [hep-ph].

[71] S. L. Glashow, D. Guadagnoli and K. Lane, Phys. Rev. Lett. 114 (2015) 091801 [arXiv:1411.0565 [hep-ph]].

[72] D. Bečirević, N. Košnik, O. Sumensari and R. Zukanovich Funchal, JHEP 1611 (2016) 035 [arXiv:1608.07583 [hep-ph]].

[73] M. Bordone, C. Cornella, J. Fuentes-Martín and G. Isidori, arXiv:1805.09328 [hep-ph]. 